\title{
Imaging Features Differentiating Vestibular Ganglion from Intracanalicular Schwannoma on Single-Sequence Non-Contrast Magnetic Resonance Imaging Study
}

Yi-Wei $\underline{\mathrm{Wu}},{ }^{1} M D, M M e d, F R C R$, Amit $\underline{\text { Karandikar}},{ }^{1} M B B S, F R C R, F A M S$, Julian PN $\underline{\mathrm{Goh}},{ }^{1} M B B S$, FRCR, Tiong Yong $\underline{\text { Tan }},{ }^{2} M B B S$, FRCR, FAMS

\begin{abstract}
Introduction: This study aimed to identify imaging features on single-sequence noncontrast magnetic resonance imaging (MRI) that differentiate the vestibular ganglion from small intracanalicular schwannomas. Materials and Methods: Ninety patients (42 men and 48 women; age: 24-87 years old) with 102 internal auditory canal (IAC) nodules (59 vestibular ganglia and 43 intracanalicular schwannoma) who underwent both singlesequence T2-weighted (T2W) non-contrast enhanced MRI studies and contrast-enhanced T1-weighted (T1W) MRI studies between May 2012 and April 2017 were evaluated. The length, width, distance to the IAC fundus and length/width ratios for all lesions were obtained and compared among groups. Diagnostic performance and cutoff values of the parameters were evaluated with receiver operating characteristics curve analysis. Area under the curve (AUC) value was calculated. Results: Vestibular ganglia have significantly smaller lengths and widths compared to intracanalicular vestibular schwannomas $(1.7 \pm 0.4$ $\mathrm{mm}$ and $1.0 \pm 0.2 \mathrm{~mm}$ versus $5.6 \pm 3.0 \mathrm{~mm}$ and $3.7 \pm 1.5 \mathrm{~mm})$. They are more fusiform in shape compared to vestibular schwannomas (length/width ratio: $1.8 \pm 0.4$ versus $1.5 \pm 0.4$ ). The lesion width demonstrated the highest diagnostic performance (AUC: 0.998). Using a cutoff width of $<1.3 \mathrm{~mm}$, the sensitivity, specificity and overall accuracy for diagnosing vestibular ganglia were $97 \%(57 / 59), 100 \%(43 / 43)$ and $98 \%(100 / 102)$, respectively. Conclusion: Vestibular ganglia may mimic intracanalicular vestibular schwannomas on a single-sequence T2W MRI. However, a fusiform shape and width $<1.3 \mathrm{~mm}$ increases confidence in the diagnosis of ganglia. Identifying the vestibular ganglion on single-sequence T2W MRI studies may obviate the need for a contrast-enhanced MRI, reducing the risks of contrast administration, additional scanning time and cost.
\end{abstract}

Ann Acad Med Singapore 2020;49:65-71

Key words: Acoustic neuroma, Internal auditory canal, Vestibulocochlear nerve

\section{Introduction}

The gold standard for diagnosis of acoustic neuroma (schwannoma) in patients of unilateral hearing loss is contrast-enhanced magnetic resonance imaging (MRI). ${ }^{1,2}$ However, limited non-contrast MRI using a single-sequence high resolution $\mathrm{T} 2$-weighted (T2W) sequence has been proposed as a cost-effective screening tool to evaluate unilateral sensorineural hearing loss. ${ }^{3,4}$ Due to improvements in software and hardware with improved spatial resolution, internal auditory canal (IAC) vestibular schwannomas as small as $2 \mathrm{~mm}$ can now be detected by MRI.,5

In our practice, patients presenting with unilateral or bilateral sensorineural hearing loss are screened with non-contrast enhanced single-sequence MRI (utilising a single high-resolution $\mathrm{T} 2 \mathrm{~W}$ sequence) to exclude a mass in the IAC and cerebellopontine angle which may need to be addressed clinically — most commonly due to vestibular schwannomas. This is performed after patients have been assessed clinically to exclude other possible causes of sensorineural hearing loss such as labyrinthitis and Meniere's disease. If any nodularity or mass is detected, a subsequent contrast-enhanced MRI ("full MRI") utilising not only the high-resolution $\mathrm{T} 2 \mathrm{~W}$ sequence, but also pre-and postcontrast T1-weighted (T1W) sequences is performed.

However, as a result of the increased resolution alluded to above, small subcentimetre nodules along the vestibular nerve are often detected by single-sequence non-contrast enhanced MRI examination. This includes normal structures

${ }^{1}$ Department of Diagnostic Radiology, Tan Tock Seng Hospital, Singapore

2Department of Diagnostic Radiology, Changi General Hospital, Singapore

Address for Correspondence: Dr Wu Yi-Wei, Department of Diagnostic Radiology, Tan Tock Seng Hospital, 11 Jalan Tan Tock Seng, Singapore 308433.

Email: wuyiwei753@gmail.com 
such as Scarpa's ganglion in the vestibular nerve, which can be seen on high quality MRI as subtle thickening or nodularity. ${ }^{6}$ Accurate differentiation of the vestibular ganglion from small subcentimetre intracanalicular vestibular schwannomas can be difficult. Although definite diagnosis can be achieved by a subsequent contrastenhanced MRI study - where vestibular ganglia show no enhancement as opposed to small vestibular schwannomas in the postcontrast $\mathrm{T} 1 \mathrm{~W}$ sequence ${ }^{7}$ - performing additional contrast-enhanced T1W MRI study requires additional scanning time and cost, as well as exposing the patient to potential risk of intravenous gadolinium administration. ${ }^{8}$

This study aimed to identify imaging features on a single-sequence non-contrast MRI study that can be used to confidently differentiate the vestibular ganglion from small intracanalicular schwannoma. This may help to reduce the total number of contrast-enhanced T1W MRI studies which are needed to differentiate between vestibular ganglia from small vestibular schwannomas.

\section{Materials and Methods \\ Patients}

Approval from the institutional review board was obtained for this retrospective study, and the requirement for informed consent was waived. From May 2012 to April 2017, there were 169 patients found to have IAC nodules/mass from single-sequence non-contrast enhanced MRI study, who subsequently underwent contrast-enhanced T1W MRI sequence. A total of 183 IAC nodules/lesions were found in the single-sequence non-contrast MRI study initially. After evaluation with contrast-enhanced T1W MRI, IAC lesions that were artefacts $(n=13)$, vascular loops $(n=6)$ and lipoma $(\mathrm{n}=1)$ were excluded. IAC lesions that could be identified in the facial $(n=1)$ or cochlear nerve $(n=7)$ were also excluded. Vestibular schwannomas that could be unequivocally diagnosed based on non-contrast T2W MRI sequence without confusion with vestibular ganglion were excluded as well. These included lesions that extended into the vestibule $(n=3)$ or cerebellopontine angle $(n=50)$.

A total of 90 patients ( 42 men and 48 women; age: $24-87$ years old; mean age: 61 years old; median age: 63 years old) with 102 IAC nodules (59 vestibular ganglia and 43 intracanalicular schwannomas) were evaluated in this study.

\section{Imaging Protocols}

All single-sequence non-contrast MRI studies $(\mathrm{n}=90)$ were performed with $1.5 \mathrm{~T}(\mathrm{n}=58$, Signa HDxt, GE Medical System, Milwaukee, United States; $\mathrm{n}=8$, Ingenia, Philips, Amsterdam, The Netherlands) or 3T ( $\mathrm{n}=24$, Trio, Siemens Healthcare, Erlangen, Germany) scanner. External phase array head coils were used for all MRIstudies ( 8 channels for GE; 15 channels for Philips; 12 channels for Siemens). Axial
T2W MRI sequences were acquired for all patients. These are 3-dimensional isotropic/isovoxel sequences that allowed multiplanar reconstruction to be performed. Imaging parameters for the single-sequence $\mathrm{T} 2 \mathrm{~W}$ examination on the 1.5T and 3T systems are summarised in Table 1. Postcontrast-enhanced axial T1W MRI sequence was available for all patients as a gold standard to differentiate the vestibular ganglion from schwannoma. With respect to contrast medium, a total of $10 \mathrm{~mL}$ of Dotarem or $5 \mathrm{~mL}$ of Gadovist was administered via hand injection intravenously prior to acquiring the contrast-enhanced images. Images were acquired using the same head coils that were used for axial $\mathrm{T} 2 \mathrm{~W}$ sequences.

\section{Imaging Interpretation and Reference Standard}

The MRI images were reviewed retrospectively by 2 radiologists (both specialised in head and neck imaging with $>20$ years of experience). IAC lesions that demonstrated enhancement in the postcontrast-enhanced T1W sequence were diagnosed as intracanalicular vestibular schwannomas, while nodules that did not enhance were considered as vestibular ganglia (Figs. 1 and 2). Any discrepancy between the 2 radiologists was settled by consensus.

All measurements were performed on the axial plane of the T2W MRI sequence, where the lesion had the largest diameter. The following parameters of the IAC lesion were measured: length (diameter along the axis of the vestibular nerve), width (diameter perpendicular to the axis of the vestibular nerve) and the distance of the lesion from the fundus of the IAC (distance between the distal margin of the lesion and the fundus) (Fig. 3). Ratios of the length/ width were calculated accordingly for all lesions.

\section{Statistical Analysis}

Chi-square test was used to evaluate whether gender, presence of hearing loss and bilaterality were associated with vestibular ganglion or schwannoma. For comparison of patient's age, lesion size, length/width ratio and distance between the lesion and IAC fundus, the student's t-test was used. For the length, width and ratio of length/width, a receiver operating characteristics (ROC) curve was done and the area under the curve (AUC) was calculated. As the role of single-sequence non-contrast enhanced MRI is to exclude as many vestibular schwannomas as possible, the cutoff values of all the parameters were determined first by the highest specificity to diagnose ganglion. If several cutoff values have the same specificity, the one with the highest sensitivity was chosen. Sensitivity, specificity, positive predictive value and negative predictive value of each imaging parameter at set cutoff value were calculated.

Statistical analysis was performed using IBM SPSS Statistics 22.0 software. A $P$ value of $<0.05$ was considered statistically significant. 
Table 1. Imaging Parameters of Single-Sequence T2-Weighted Examination

\begin{tabular}{|c|c|c|c|c|c|c|c|c|}
\hline System & $\begin{array}{l}\text { Repetition } \\
\text { Time (ms) }\end{array}$ & $\begin{array}{c}\text { Echo Time } \\
\text { (ms) }\end{array}$ & $\begin{array}{c}\text { Slice Thickness } \\
(\mathrm{mm})\end{array}$ & Matrix & $\begin{array}{c}\text { Voxel Size } \\
\text { (mm) }\end{array}$ & $\begin{array}{l}\text { Field of View } \\
\qquad(\mathrm{cm})\end{array}$ & Flip Angle $\left(^{\circ}\right)$ & $\begin{array}{l}\text { Number of } \\
\text { Excitations }\end{array}$ \\
\hline $\begin{array}{l}1.5 \mathrm{~T} \text { GE } \\
\text { (FIESTA) }\end{array}$ & 5.9 & $2.6-122$ & 0.8 & $256 \times 256$ & 0.35 & 18 & 65 & 3 \\
\hline $\begin{array}{l}\text { 1.5T Philips } \\
\text { (DRIVE) }\end{array}$ & 1500 & 125 & 0.8 & $248 \times 247$ & 0.41 & 16 & 90 & 1 \\
\hline $\begin{array}{l}\text { 3T Siemens } \\
\text { (SPACE) }\end{array}$ & 1000 & 135 & 0.5 & $380 \times 384$ & 0.26 & 20 & 120 & 1.6 \\
\hline
\end{tabular}

DRIVE: Driven equilibrium; FIESTA: Fast Imaging Employing Steady-State Acquisition; SPACE: Sampling Perfection with Application Optimised Contrasts Using Different Flip Angle Evolution

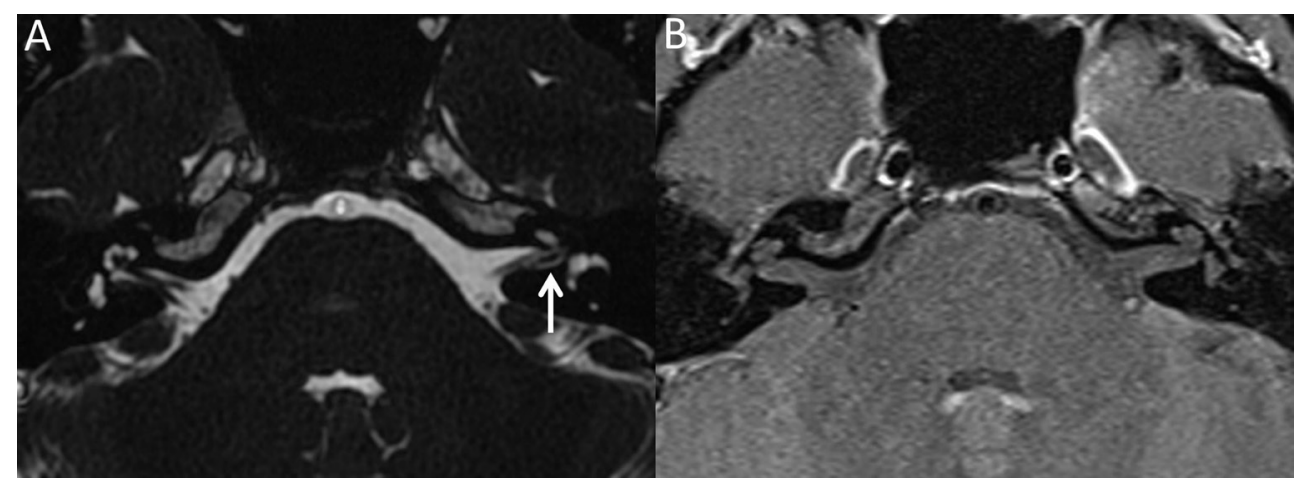

Fig. 1. Vestibular ganglion in a patient with left sensorineural hearing loss. Axial T2-weighted magnetic resonance image (MRI) of the internal auditory canal (A) showed a small fusiform lesion along the left superior vestibular nerve. Subsequent contrast-enhanced MRI in T1-weighted sequence (B) showed no enhancement in the left internal auditory canal that confirmed the diagnosis of vestibular ganglion.

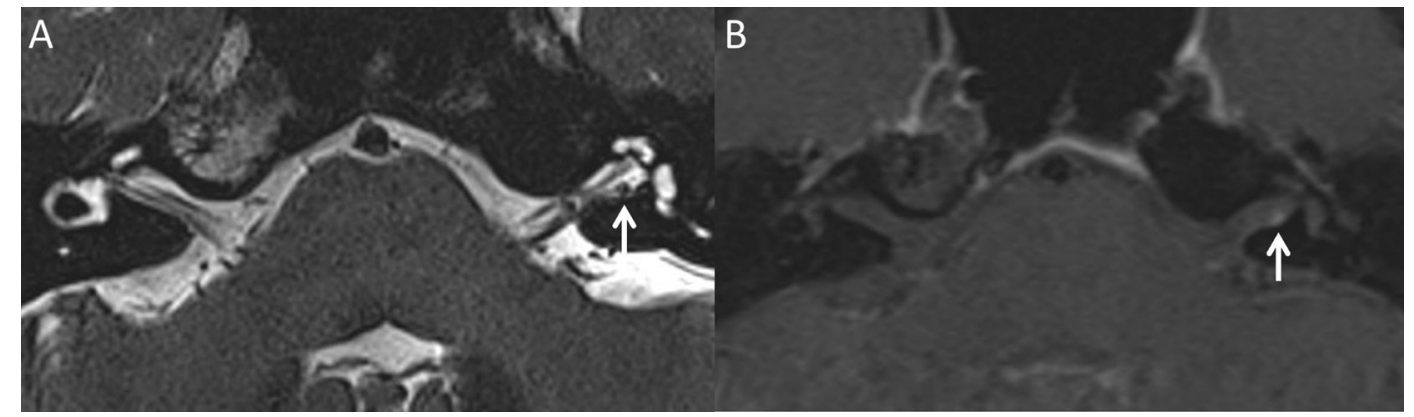

Fig. 2. Intracanalicular schwannoma in a patient with left sensorineural hearing loss. Axial T2-weighted magnetic resonance image (MRI) of the internal auditory canal (A) showed a small rounded lesion along the left inferior vestibular nerve. Diagnosis of intracanalicular schwannoma was made in the subsequent contrast-enhanced MRI in T1-weighted sequence as the lesion demonstrated enhancement (B).

\section{Results}

\section{Patient and IAC Lesion Characteristics}

A total of 90 patients (48 in the vestibular ganglion group; 43 in intracanalicular schwannoma group; 1 in both groups) were included in this study. The demographic and clinical data of the 2 groups are summarised in Table 2.

The side of nodules did not show significant difference $(P$ $=0.7$ ) among vestibular ganglion (left/right ratio: $35 / 24)$ and intracanalicular schwannoma (left/right ratio: 24/19) groups. The location of the vestibular ganglion was detected in the inferior division of the vestibular nerve $(76 \%, 45 / 59)$ and in the superior division of the vestibular nerve $(24 \%, 14 / 59)$.
Similarly, 21\% (9/43) of the intracanalicular schwannomas was seen in the inferior division of the vestibular nerve and $14 \%(6 / 43)$ was detected in the superior division of the vestibular nerve. The location of the other intracanalicular schwannomas $(65 \%, 28 / 43)$ was undetermined due to large size. The percentage of vestibular schwannoma observed on the side of the presenting symptom was $88 \%(38 / 43)$, while 58\% (34/59) was found for vestibular ganglion $(P=$ $0.001)$. In the group of patients with ganglia, $23 \%(11 / 48)$ presented with bilateral vestibular ganglia. There was no significant size difference between bilateral vestibular ganglia and all of them were located in the same nerve. 


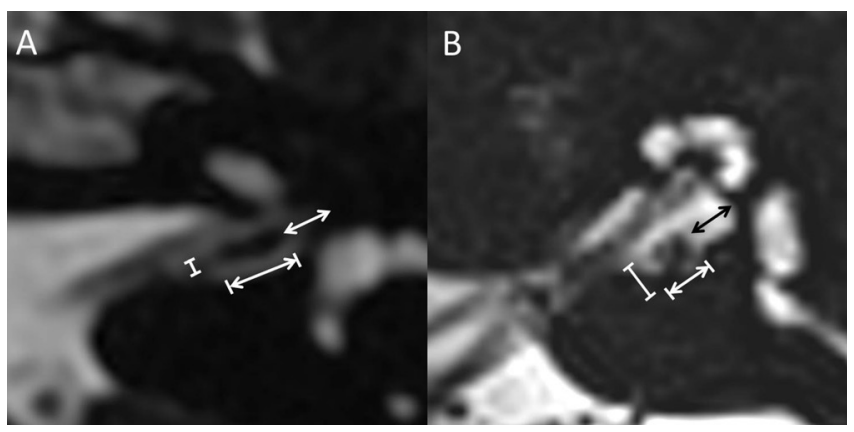

Fig. 3. Measurements of vestibular ganglion and intracanalicular schwannoma. Axial T2-weighted images showed vestibular ganglion (A) and intracanalicular vestibular schwannoma (B). The length $(\mid \leftrightarrow I)$, width (I-I) and distance to the fundus of the internal auditory canal $(\leftrightarrow)$ of the ganglion measured $3.0 \mathrm{~mm}, 0.7$ $\mathrm{mm}$ and $2.4 \mathrm{~mm}$, respectively (A). The ratio of the length and width of the ganglion is 4.3. Similarly, the length, width and distance to the fundus of the intracanalicular schwannoma measured $2.0 \mathrm{~mm}, 1.7 \mathrm{~mm}$ and $2.2 \mathrm{~mm}$, respectively (B). The ratio of the length and width of the schwannoma is 1.2.

Table 2. Demographic and Clinical Data

\begin{tabular}{lccc}
\hline Variable & $\begin{array}{c}\text { Patients With } \\
\text { Ganglion } \\
\mathbf{n}=\mathbf{4 8}\end{array}$ & $\begin{array}{c}\text { Patients With } \\
\text { Schwannoma } \\
\mathbf{n}=\mathbf{4 3}\end{array}$ & $\begin{array}{c}\boldsymbol{P} \\
\text { Value }\end{array}$ \\
\hline $\begin{array}{l}\text { Age in years } \\
(\text { mean } \pm \text { SD) }\end{array}$ & $58.6 \pm 15.1$ & $63.3 \pm 9.9$ & 0.086 \\
$\begin{array}{l}\text { Gender (male/ } \\
\text { female ratio) }\end{array}$ & $20 / 28$ & $23 / 20$ & 0.26 \\
$\begin{array}{l}\text { Hearing loss as } \\
\text { indication }(\%)\end{array}$ & $75(36 / 48)$ & $88(38 / 43)$ & 0.1 \\
$\begin{array}{l}\text { Patients with } \\
\text { bilateral ganglia } \\
\text { or bilateral } \\
\text { schwannomas }(\%)\end{array}$ & $23(11 / 48)$ & $0(0 / 43)$ & $<0.001$ \\
\hline
\end{tabular}

SD: Standard deviation

\section{Imaging Features}

The statistical significance of imaging features that differentiate vestibular ganglion and intracanalicular schwannoma is summarised in Table 3.

\section{Cutoff Values of Imaging Features and Diagnostic Performance}

The ROC curves of length, width and ratio are presented in Figures 4 and 5. The AUC values for length, width and ratio were $0.974,0.998$ and 0.766 , respectively.

The cutoff values of length, width and ratio for diagnosing vestibular ganglion were set at $<1.9 \mathrm{~mm},<1.3 \mathrm{~mm}$ and $>2.3$, respectively. By applying the cutoff value of length $<1.9 \mathrm{~mm}$, the sensitivity, specificity and overall accuracy of diagnosing vestibular ganglion were $66 \%$ (39/59), 100\% $(43 / 43)$ and $80 \%(82 / 102)$, respectively. By applying the cutoff value of width $<1.3 \mathrm{~mm}$, the sensitivity, specificity
Table 3. Imaging Features of Ganglion and Schwannoma

\begin{tabular}{lccc}
\hline Variable & $\begin{array}{c}\text { Ganglion* }^{*} \\
\text { Mean } \pm \text { SD }\end{array}$ & $\begin{array}{c}\text { Schwannoma } \\
\text { Mean } \pm \text { SD }\end{array}$ & $\begin{array}{c}\boldsymbol{P} \\
\text { Value }\end{array}$ \\
\hline Length $(\mathrm{mm})$ & $1.7 \pm 0.4$ & $5.6 \pm 3.0$ & $<0.001$ \\
\hline Width $(\mathrm{mm})$ & $1.0 \pm 0.2$ & $3.7 \pm 1.5$ & $<0.001$ \\
$\begin{array}{l}\text { Length/width } \\
\text { ratio }\end{array}$ & $1.8 \pm 0.4$ & $1.5 \pm 0.4$ & $<0.001$ \\
$\begin{array}{l}\text { Distance from } \\
\text { fundus (mm) }\end{array}$ & $2.2 \pm 0.7$ & $2.0 \pm 1.4$ & 0.23 \\
\hline
\end{tabular}

SD: Standard deviation

${ }^{*} \mathrm{n}=59$.

${ }^{\dagger} \mathrm{n}=43$.

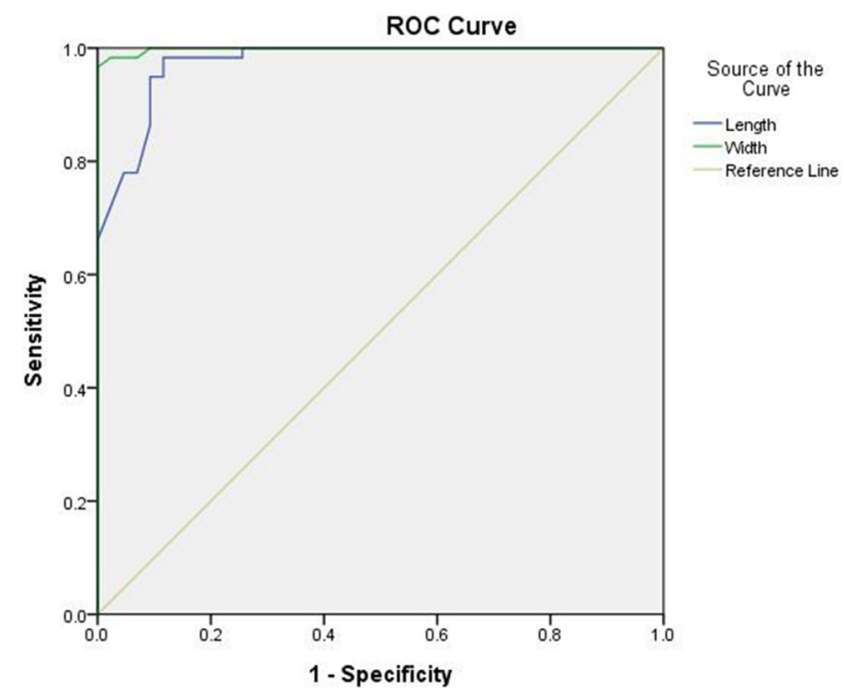

Fig. 4. Receiver operating characteristics curve of length and width in diagnosing vestibular ganglion.

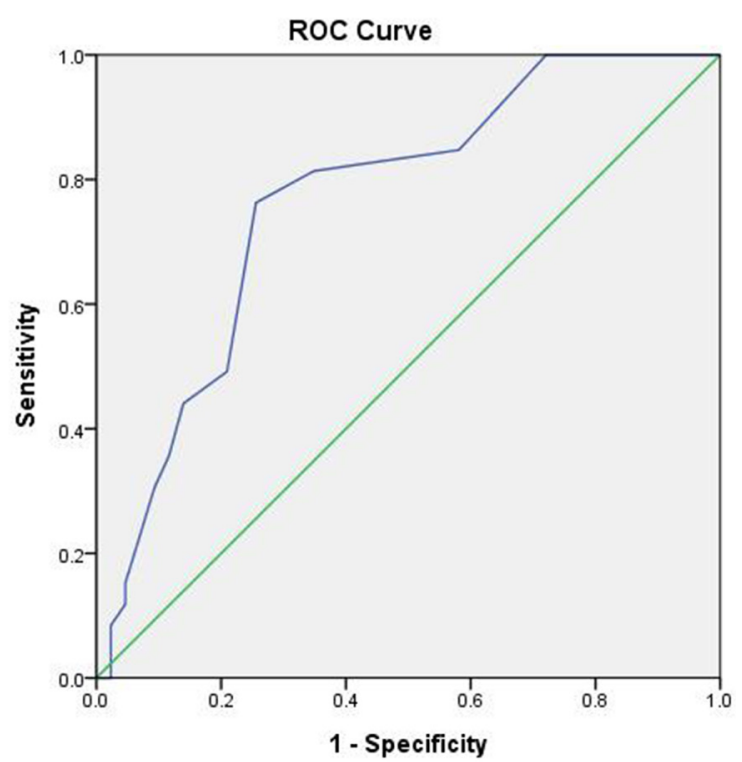

Fig. 5. Receiver operating characteristics curve of length/width ratio in diagnosing vestibular ganglion. 
and overall accuracy of diagnosing vestibular ganglion were $97 \%(57 / 59), 100 \%(43 / 43)$ and $98 \%$ (100/102), respectively. By applying the cutoff value of ratio $>2.3$, the sensitivity, specificity and overall accuracy of diagnosing vestibular ganglion were $8 \%(5 / 59), 98 \%(43 / 43)$ and $49 \%(50 / 102)$, respectively. The diagnostic performance of these parameters for diagnosing vestibular ganglion is summarised in Table 4.

By applying the cutoff value of width $<1.3 \mathrm{~mm}$ to diagnose ganglion, 2 vestibular ganglia were classified as schwannomas due to their larger size $(1.3 \mathrm{~mm}$ and 1.6 $\mathrm{mm})$. Similarly, these 2 nodules would be falsely classified as schwannoma if the cutoff values of length $(<1.9 \mathrm{~mm})$ and ratio $(>2.3)$ are used (as the lengths of these 2 nodules were $2.1 \mathrm{~mm}$ and $2.5 \mathrm{~mm}$, respectively, and length/width ratio of both nodules was 1.6). The side of the patient's symptoms corresponded to the side of these 2 vestibular ganglia. One ganglion had similar nodule of smaller size on the contralateral side.

\section{Discussion}

The vestibulocochlear nerve (cranial nerve VIII) traverses the IAC and cerebellopontine angle (Fig. 1), extending from the cochlea and vestibule to the brainstem. The nerve comprises the superior and vestibular nerves, which receive afferents from the vestibule and semicircular canals; and the cochlear nerve, which receives afferents from the cochlea itself.

The vestibular ganglion, also known as Scarpa's ganglion, is the focal enlargement of the vestibular nerve within the IAC that contains cell bodies of the bipolar primary neurons. ${ }^{9}, 10$ These are occasionally demonstrated on high resolution T2W MRI sequence, mimicking small schwannomas. Accurate imaging diagnosis to differentiate vestibular ganglion and small schwannoma is therefore essential as microsurgery and stereotactic radiation can be used to treat symptomatic patients with intracanalicular schwannoma. ${ }^{11-13}$

This study sought to identify imaging features in singlesequence non-contrast MRI T2W studies that can be reliably used to differentiate the vestibular ganglion from intracanalicular vestibular schwannoma, so as to reduce the number of patients having further follow-up contrastenhanced T1W MRI studies. While the contrast-enhanced MRI is the gold standard, the rationale for performing a single-sequence study is multifold. Firstly, patients would have already been assessed for other causes of sensorineural hearing loss by the otolaryngologist; many of these causes are clinically apparent (e.g. labyrinthitis, Meniere's disease). Secondly, with the increasing emphasis on maintaining reasonable healthcare costs and the efficient utilisation of imaging resources, a single-sequence examination that can detect the presence of a mass in the IAC and/ or cerebellopontine angle is a more cost-efficient use of imaging technology. This is relevant both in terms of time expended (approximately 4-5 minutes for a singlesequence examination versus approximately 30 minutes for a contrast-enhanced examination) and actual costs (approximately $\$ 180$ and $\$ 390$ for subsidised and paying patients, respectively, for the single-sequence examination; and $\$ 570$ and $\$ 1220$, respectively, for the full examination). Also, given the low incidence of vestibular schwannomas (approximately 4\%), ${ }^{4}$ subjecting patients to a full contrastenhanced examination to identify a small number of schwannomas leads to unnecessary costs.

In our study, most of the vestibular ganglia presented as tiny nodules ( $<3 \mathrm{~mm}$ for both length and width) along the vestibular nerve with fusiform shape along the axis of the nerve (average length to width ratio: 1.8). The location of the ganglion was most commonly seen in the inferior division of the vestibular nerve $(76 \%)$ and approximately $2 \mathrm{~mm}$ from the fundus of the IAC. These morphological features observed in the single-sequence non-contrast MRI are compatible with findings in anatomical study. ${ }^{9}$ To our knowledge, this is the first study that attempted differentiation of intracanalicular schwannomas from vestibular ganglia. We also looked at imaging features of a vestibular ganglion.

Vestibular schwannoma most commonly originates from the vestibular ganglion in the distal vestibular nerve. ${ }^{1}$

Table 4. Diagnostic Performance for Ganglion

\begin{tabular}{|c|c|c|c|c|c|c|c|c|c|}
\hline Variable & $\begin{array}{c}\text { Sensitivity } \\
(\%)\end{array}$ & $95 \%$ CI & $\begin{array}{l}\text { Specificity } \\
(\%)\end{array}$ & $95 \% \mathrm{CI}$ & PPV (\%) & $95 \% \mathrm{CI}$ & NPV (\%) & $95 \% \mathrm{CI}$ & $\begin{array}{c}\text { Accuracy } \\
(\%)\end{array}$ \\
\hline $\begin{array}{l}\text { Length } \\
(<1.9 \mathrm{~mm})\end{array}$ & $66(39 / 59)$ & $53-78$ & $100(43 / 43)$ & $90-100$ & $100(39 / 39)$ & $89-100$ & $68(43 / 64)$ & $55-79$ & $80(82 / 102)$ \\
\hline $\begin{array}{l}\text { Width } \\
(<1.3 \mathrm{~mm})\end{array}$ & $97(57 / 59)$ & $87-99$ & $100(43 / 43)$ & $90-100$ & $100(57 / 57)$ & $92-100$ & $96(43 / 45)$ & $84-99$ & $98(100 / 102)$ \\
\hline $\begin{array}{l}\text { Ratio of length/ } \\
\text { width }(>2.3)\end{array}$ & $8(5 / 59)$ & $3-19$ & $98(42 / 43)$ & $86-100$ & $89(8 / 9)$ & $36-99$ & $45(42 / 93)$ & $34-54$ & $49(50 / 102)$ \\
\hline
\end{tabular}

CI: Confidence interval; NPV: Negative predictive value; PPV: Positive predictive value 
Therefore, small intracanalicular vestibular schwannomas may share similar imaging findings with vestibular ganglia. In this study, we found that both vestibular ganglia and schwannomas are more commonly detected in the inferior division of the vestibular nerves and usually $2 \mathrm{~mm}$ away from the IAC fundus. However, the size and shape were the imaging features that were helpful to differentiate one from the other. In our study, the vestibular ganglion was significantly smaller in size (compared to vestibular schwannoma) and more fusiform in shape (average length to width radio: 1.8) compared to schwannoma that is more spherical in shape (average length to width ratio: 1.5). In this study, using a width $<1.3 \mathrm{~mm}$ as a cutoff to diagnose vestibular ganglion can reliably exclude all the intracanalicular vestibular schwannomas and 57 out of 59 vestibular ganglia were accurately diagnosed based on single-sequence non-contrast MRI. If this cutoff value is used, 51\% (46/90) of contrast-enhanced T1W MRI studies in our study population could have been avoided. This is particularly helpful to reduce the number of contrastenhanced T1W MRI examinations that require additional scanning time and cost.

In our study, $23 \%$ of patients with vestibular ganglia presented with symmetrical bilateral IAC nodules of similar imaging features. We found that length and width difference between bilateral ganglia of these patients was $<1 \mathrm{~mm}$ and the difference of distance from the nodule to IAC fundus ranged from $0.2-1.3 \mathrm{~mm}$. On the other hand, none of the patients in the schwannoma group presented with bilateral intracanalicular schwannomas. Bilateral vestibular schwannomas are extremely rare and the entity is known to be associated with neurofibromatosis type 2(NF2) which has an incidence of 1 in 25,000-1 in 40,000. ${ }^{14}$ Elderly patients may develop incidental bilateral vestibular schwannomas with even lower incidence $\left(1\right.$ in 2,000,000) ${ }^{15}$ In the absence of known NF2, small bilateral symmetrical IAC nodules can be considered as a useful feature to differentiate vestibular ganglion from intracanalicular vestibular schwannoma.

The percentage of vestibular schwannoma that corresponded to the side of symptom ( $88 \%$ ) was significantly higher compared to vestibular ganglion (58\%) in this study. However, correspondence to the side of symptom alone cannot reliably distinguish one from the other. Interestingly, there were few intracanalicular schwannomas $(12 \%, 5 / 43)$ in this study that were detected on the contralateral side of presenting symptom. These schwannomas were small in size (length of lesion ranging from $2-5 \mathrm{~mm}$ ) and were most likely incidental findings.

Our study has a few limitations. First, pathological diagnosis of vestibular schwannoma was only available for a small number of cases. Most of the diagnoses were made based on the "gold standard" of contrast-enhanced T1W
MRI. However, this is reflective of current clinical practice, where only lesions that cause significant symptoms warrant surgery and histological diagnosis. Second, selection bias was one of the potential limitations for this retrospective study, as vestibular ganglia that underwent both singlesequence non-contrast MRI and contrast-enhanced T1W MRI are likely larger nodules. However, the result of the study is unlikely to be affected if smaller nodules are also included. Third, different scanners and imaging protocols with different spatial resolution were used in this study. Nevertheless, heterogeneity of these factors reflects the nature of routine practice.

\section{Conclusion}

Bilateral symmetrical IAC nodules found in the singlesequence non-contrast MRI that are fusiform in shape (average length to width ratio: 1.8) and small in size (width $<1.3 \mathrm{~mm}$ ) are likely to be vestibular ganglia rather than vestibular schwannomas. Key differences between vestibular ganglion and intracanalicular schwannoma on single-sequence non-contrast MRI are summarised in Table 5. Furthermore, using a width cutoff of $<1.3 \mathrm{~mm}$ of the IAC nodule further increases confidence in diagnosing vestibular ganglion. However, we recognise that although, there were no intracanalicular schwannomas $<1.3 \mathrm{~mm}$ of width in our study, this remains a possibility. We propose that fusiform IAC nodules seen approximately $2 \mathrm{~mm}$ from the fundus with width $<1.3 \mathrm{~mm}$ on single-sequence non-contrast MRI should be diagnosed as ganglion without further need for assessment by contrast-enhanced T1W MRI (unless there is further deterioration of symptom on the ipsilateral side on clinical follow-up). This will help to reduce risks of contrast administration, additional scanning time and cost.

Table 5. Key Differences Between Vestibular Ganglion and Intracanalicular Schwannoma

\begin{tabular}{lccc}
\hline Variable & Size & Shape & Distribution \\
\hline Ganglion & $\begin{array}{c}\text { Smaller in size } \\
(\text { width }<1.3\end{array}$ & Fusiform & $\begin{array}{c}\text { Commonly } \\
\text { distributed in } \\
\text { bilateral IACs }\end{array}$ \\
Schwannoma & Larger in size & Rounded & $\begin{array}{c}\text { Uncommon, } \\
\text { unless the } \\
\text { patient is }\end{array}$ \\
& & known to have \\
& & NF2 \\
\hline
\end{tabular}

IAC: Internal auditory canal; NF2: Neurofibromatosis type 2 


\section{REFERENCES}

1. Lin EP, Crane BT. The management and imaging of vestibular schwannomas. AJNR Am J Neuroradiol 2017;38:2034-43.

2. Abele TA, Besachio DA, Quigley EP, Gurgel RK, Shelton C, Harnsberger HR, et al. Diagnostic accuracy of screening MR imaging using unenhanced axial CISS and coronal T2WI for detection of small internal auditory canal lesions. AJNR Am J Neuroradiol 2014;35:2366-70.

3. Crowson MG, Rocke DJ, Hoang JK, Weissman JL, Kaylie DM. Costeffectiveness analysis of a non-contrast screening MRI protocol for vestibular schwannoma in patients with asymmetric sensorineural hearing loss. Neuroradiology 2017;59:727-36.

4. Tan TY, Teh HS. Contrast-enhanced magnetic resonance imaging of the internal auditory canals and posterior fossa. Ann Acad Med Singapore 1998;27:168-72.

5. Fortnum H, O'Neill C, Taylor R, Lenthall R, Nikolopoulos T, Lightfoot $\mathrm{G}$, et al. The role of magnetic resonance imaging in the identification of suspected acoustic neuroma: a systematic review of clinical and cost effectiveness and natural history. Health Technol Assess 2009;13:iii-iv, ix-xi, 1-154.

6. Casselman J, Mermuys K, Delanote J, Ghekiere J, Coenegrachts K. MRI of the cranial nerves - more than meets the eye: technical considerations and advanced anatomy. Neuroimaging Clin N Am 2008;18:197-231, preceding $\mathrm{X}$.
7. Verbist BM. Imaging of sensorineural hearing loss: a pattern-based approach to diseases of the inner ear and cerebellopontine angle. Insights Imaging 2012;3:139-53.

8. Gulani V, Calamante F, Shellock FG, Kanal E, Reeder SB, International Society for Magnetic Resonance in M. Gadolinium deposition in the brain: summary of evidence and recommendations. Lancet Neurol 2017;16:564-70.

9. Bergstrom B. Morphology of the vestibular nerve. I. Anatomical studies of the vestibular nerve in man. Acta Otolaryngol 1973;76:162-72.

10. Park JJ, Tang Y, Lopez I, Ishiyama A. Age-related change in the number of neurons in the human vestibular ganglion. J Comp Neurol 2001;431:437-43.

11. Quesnel AM, McKenna MJ. Current strategies in management of intracanalicular vestibular schwannoma. Curr Opin Otolaryngol Head Neck Surg 2011;19:335-40.

12. Guerin C, Sampath P, Long DM. Acoustic neuroma: outcome of surgical resection and study on the anatomy of facial and cochlear nerves. Ann Acad Med Singapore 1999;28:402-8.

13. Brackmann DE. Acoustic neuroma: surgical approaches and complications. Ann Acad Med Singapore 1991;20:674-9.

14. Lu-Emerson C, Plotkin SR. The neurofibromatoses. Part 2: NF2 and schwannomatosis. Rev Neurol Dis 2009;6:E81-6.

15. Evans DG, Freeman S, Gokhale C, Wallace A, Lloyd SK, Axon P, et al. Bilateral vestibular schwannomas in older patients: NF2 or chance? J Med Genet 2015;52:422-4. 